\title{
Mireille CANALis-DuRAND \\ REINHARD SCHÄFKE \\ Divergence and summability of normal \\ forms of systems of differential equations with nilpotent linear part
}

Annales de la faculté des sciences de Toulouse $\sigma^{e}$ série, tome 13, $\mathrm{n}^{\mathrm{o}} 4$ (2004), p. 493-513

<http://www.numdam.org/item?id=AFST_2004_6_13_4_493_0>

(C) Université Paul Sabatier, 2004, tous droits réservés.

L'accès aux archives de la revue «Annales de la faculté des sciences de Toulouse » (http://picard.ups-tlse.fr/ annales/) implique l'accord avec les conditions générales d'utilisation (http://www.numdam.org/conditions). Toute utilisation commerciale ou impression systématique est constitutive d'une infraction pénale. Toute copie ou impression de ce fichier doit contenir la présente mention de copyright.

\section{NumDam}

Article numérisé dans le cadre du programme Numérisation de documents anciens mathématiques http://www.numdam.org/ 


\title{
Divergence and summability of normal forms of systems of differential equations with nilpotent linear part
}

\author{
Mireille Canalis-Durand ${ }^{(1)}$, Reinhard Schäfke $^{(2)}$
}

Abstract. - We consider the following system of differential equations

$$
\dot{x}=2 y+2 x \Delta(x, y), \quad \dot{y}=3 x^{2}+3 y \Delta(x, y),
$$

where $\Delta \in \mathbb{C}\{x, y\}, \Delta(0,0)=0$. The above system is a prenormal form of a generic perturbation of the hamiltonian system $\dot{x}=2 y, \dot{y}=3 x^{2}$. Substituting $x$ by $x U(x, y)^{2}$ and $y$ by $y U(x, y)^{3}$ where $U(x, y)$ is a formal power series with $U(0,0)=1,(\Delta)$ can be transformed into a formal normal form

$$
\dot{x}=2 y+2 x \Delta^{*}, \quad \dot{y}=3 x^{2}+3 y \Delta^{*},
$$

where $\Delta^{*}=A_{0}(h)+x A_{1}(h), h=y^{2}-x^{3}$, and $A_{0}(h)=A_{0 k} h^{k}+\ldots$, $A_{1}(h)=A_{1 l} h^{l}+\ldots$ are formal power series with $A_{0 k}, A_{1 l} \neq 0$ ([Lo99]).

In the present work, we discuss the Gevrey order and summability of the formal power series $A_{0}, A_{1}$ and $U$ as a function of the above valuations $k$ and $l$ of the series $A_{0}$ and $A_{1}$. Here, we distinguish two cases: in the first case, $l$ is smaller than $k$, in the second $k \leqslant l$. The first case includes the non-degenerate case where $\Delta=\alpha x+\ldots, \alpha \neq 0$; all the others are degenerate. In this work, we present the following results:

In the first case, $A_{1}$ can be reduced to $A_{1 l} h^{l}$ and the power series $U(x, y)=\sum_{r, s} b_{r s} x^{r} y^{s}$ and $A_{0}(h)=\sum_{m \geqslant k} A_{0 m} h^{m}$ are Gevrey of order $\alpha=1 /(6 l+1)$ in the weighted degree; i.e. there exist $K, A>0$ such that for all $r, s, m$

$\left|A_{m}\right| \leqslant K A^{6 m \alpha} \Gamma(6 m \alpha+1), \quad\left|b_{r s}\right| \leqslant K A^{(2 r+3 s) \alpha} \Gamma((2 r+3 s) \alpha+1)$. Moreover, the power series $A_{0}\left(t^{6}\right)$ is summable and generically, the above Gevrey order is optimal. In the second case, $A_{0}$ can be reduced to $A_{0 k} h^{k}$ and $U(x, y)$ and $A_{1}(h)$ are Gevrey of order $1 /(3 k+3 l)$ in the weighted degree. Moreover, the power series $A_{1}\left(t^{6}\right)$ is summable and generically, the above Gevrey order is optimal.

In the non-degenerate case, we also detail the type $A$ of the series.

(1) GREQAM, Université d'Aix-Marseille III, 13002 Marseille.

E-mail: canalis@ehess.univ-mrs.fr

(2) IRMA, Université Louis Pasteur, 67084 Strasbourg Cedex.

E-mail: schaefke@math.u-strasbg.fr 
RÉSUMÉ. - On considère des formes prénormales associées à des perturbations génériques du système $\dot{x}=2 y, \dot{y}=3 x^{2}$. Il est connu qu'elles admettent une forme normale formelle $\dot{x}=2 y+2 x \Delta^{*}, \dot{y}=3 x^{2}+3 y \Delta^{*}$, où $\Delta^{*}=A_{0}(h)+x A_{1}(h)$ avec $h=y^{2}-x^{3}$ ([Loray, 1999]). Nous démontrons que $A_{0}, A_{1}$ et les transformations normalisantes sont divergentes, mais $k$-sommables. L'entier $k$ dépend des premiers termes non nuls de $A_{0}$ et $A_{1}$.

\section{Introduction}

We consider the system of differential equations:

$$
\left\{\begin{array}{l}
\dot{x}=2 y+2 x \Delta(x, y) \\
\dot{y}=3 x^{2}+3 y \Delta(x, y)
\end{array}\right.
$$

where $\Delta \in \mathbb{C}\{x, y\}$, i.e. a convergent power series of $y, \Delta(0,0)=0$ and $\cdot=\frac{d}{d z}$.

Remark 1.1. - The above system is a prenormal form of a generic perturbation of the hamiltonian system

$$
\left\{\begin{array}{l}
\dot{x}=2 y \\
\dot{y}=3 x^{2}
\end{array} .\right.
$$

D. Cerveau and R. Moussu ([CM88]) have shown that this prenormal form can always be reached by a convergent transformation. F. Loray ([Lo99]) showed that $(\Delta)$ can be transformed into a system $(\tilde{\Delta})$ - of the same form, but with $\Delta$ replaced by $\tilde{\Delta}$ - by a substitution

$$
\begin{gathered}
x \leftarrow x U^{2}(x, y) \\
y \leftarrow y U^{3}(x, y) \\
d z \leftarrow U(x, y)^{-1} d z
\end{gathered}
$$

where $U(x, y)$ is a convergent or formal power series with $U(0,0)=1$. Thus $(\Delta)$ is transformed into

$$
\left\{\begin{array}{l}
\dot{x}=2 y+2 x \tilde{\Delta}(x, y) \\
\dot{y}=3 x^{2}+3 y \tilde{\Delta}(x, y)
\end{array}\right.
$$

The transformation equation connecting $\Delta, \tilde{\Delta}$ and $U$ is

$$
\left(U+2 x U_{x}+3 y U_{y}\right) \tilde{\Delta}=\Delta\left(x U^{2}, y U^{3}\right)-2 y U_{x}-3 x^{2} U_{y}
$$

where subscripts ${ }_{x}$ etc denote partial derivatives. 
Divergence and summability of normal forms of systems of differential equations

If $U$ is convergent, we say that $(\Delta)$ is analytically equivalent to $(\tilde{\Delta})$. If $U$ is only a formal power series, we say that $(\Delta)$ is formally equivalent to $(\tilde{\Delta})$.

It is natural to ask for the "simplest" $(\tilde{\Delta})$ equivalent to a given $(\Delta)$, i.e. for a normal form which will be denoted by $\left(\Delta^{*}\right)$.

TheOREM 1.2 ([Lo99]). - Given $\Delta \in \mathbb{C}\{x, y\}, \Delta(0,0)=0$, there is a unique formal power series $U(x, y)=1+\ldots$ transforming $(\Delta)$ into its formal normal form $\left(\Delta^{*}\right), \Delta^{*}=A_{0}(h)+x A_{1}(h)$, where $h=y^{2}-x^{3}$ and $A_{0}, A_{1} \in \mathbb{C}[[h]]$. The series $\Delta$ and $\Delta^{*}$ are related by (1.2). More precisely, $\Delta^{*}$ is in one of the following form $\Delta^{*}=0, \Delta^{*}=a_{l} h^{l}, \Delta^{*}=a_{k} x h^{k}$, $\Delta^{*}=a_{l} h^{l}+x A_{1}(h)$ or $\Delta^{*}=A_{0}(h)+a_{k} x h^{k} ;$ in the forth case, $A_{1}(h)$ contains no terms of lower order than $h^{l}$, in the last case $A_{0}(h)$ contains only terms of higher order than $h^{k}$.

As in the resonant saddle case [MR83], the question of convergence of this normal form and the normalizing transformation arises. There, the normal form was polynomial, but the normalizing transformation was divergent, but nevertheless summable. Moreover, it is known that if two convergent systems $(\Delta)$ and $(\tilde{\Delta})$ are formally equivalent, then according to a theorem of [CM88], they are analytically equivalent, i.e. the normalizing transformation converges if the series $\Delta^{*}$ is convergent.

With the background of these statements, R. Moussu, F. Loray, J.-F. Mattei and J.-P. Ramis asked the question about the convergence/divergence of the normal form $\Delta^{*}=A_{0}(h)+x A_{1}(h)$ and the normalizing transformation $U$.

We distinguish two cases: the non-degenerate case where $\Delta=\alpha x+\ldots$, $\alpha \neq 0$ and the degenerate case where $\alpha=0$. The non-degenerate case will be treated in some detail below and in section 2, the degenerate case somewhat less detailed below and in section 3 .

In [CMT01], the first 30 terms of $A_{0}(h)$ were calculated for several examples in the non-degenerate case, using MAPLE and resources of the computer algebra center MEDICIS. The authors used a "pattern recognition algorithm" for the growth rate of the coefficients of the power series $A_{0}$. The procedure includes several steps (numerical analysis of the coefficients, cut-off asymptotic ([RS96]), method of least squares) to determine the con- 
stants of the Gevrey character ${ }^{1}$ of the power series. They obtained numerical evidence that $A_{0}(h)$ is generically divergent, but of Gevrey order 6 and type $A=0.18 \ldots, A_{0}$ considered as a power series in $h$.

A similar approach had been used earlier in the field of singular perturbations: in 1985, the first fifty coefficients of the formal solutions of the forced Van der Pol equation were calculated ([CDG89]) and these results suggested the possible Gevrey character of the formal power series. Five years later, a proof of the Gevrey character of the formal solution was given in ([Ca91]). As a consequence, the existence of so called "canard" solutions could also be established using Borel-Laplace summation and generalized in [CRSSO0].

For the non-degenerate case the results are summarized in the theorem below. For convenience, we introduce the weighted degree by $\operatorname{deg}(x)=2$; $\operatorname{deg}(y)=3$ so $\operatorname{deg}(h)=\operatorname{deg}\left(y^{2}-x^{3}\right)=6$.

THEOREM 1.3. - With notations as above and when $\Delta=x+\ldots$, then $A_{1}=1$ and the power series $U(x, y)=\sum_{r, s} b_{r s} x^{r} y^{s}$ and $A_{0}(h)=\sum_{m \geqslant 1} A_{m} h^{m}$ are Gevrey 1 in the weighted degree; i.e. there exist $K, A>0$ such that for all $k, l, m$

$$
\left|A_{m}\right| \leqslant K A^{6 m}(6 m) !, \quad\left|b_{r s}\right| \leqslant K A^{2 r+3 s}(2 r+3 s) !,
$$

where the type $A=0.1844 \pm 0.0001$.

The power series $A_{0}\left(t^{6}\right)$ is 1-summable if $\arg t$ is not congruent to $\frac{\pi}{6}$ modulo $\frac{\pi}{3}$. Finally, there exists a non-zero analytic function $Q$ such that $Q(\Delta) \neq 0$ implies that $U$ and $\Delta^{*}=x+A_{0}(h)$ are divergent and the above type is optimal.

The above theorem was presented in ([CS03]); a complete proof will be given in a subsequent article. In the present work, a sketch of the proof in 6 steps is given in section 2 :

- Step 1: Transformation of the coordinates (elliptic functions).

- Step 2: The Borel plane.

(1) Definition ([Gev18, Ram78, RS96]): Let $k, A$ two positive numbers. A formal power power series $\widehat{f}(t)=\sum_{n \geqslant 0} a_{n} t^{n} \in \mathbb{C}[[t]]$ is said to be Gevrey of order $1 / p$ and type $A$, if there exist two nonnegative numbers $C$ and $\rho$ such that

$$
\forall n, n \geqslant 0,\left|a_{n}\right| \leqslant C A^{n / p} \Gamma(\rho+n / p)
$$


Divergence and summability of normal forms of systems of differential equations

- Step 3: The linearized problem.

- Step 4: Zeroes of the function $\mathbf{R}(\tau)=\frac{1}{2 \pi i} \int_{\mathcal{H}} e^{\tau I(s) / 2} d s$.

- Step 5: The non-linear problem.

- Step 6: Divergence.

On the last step, the origin and the nature of the function $Q$ will become clear.

As also the degenerate cases fall into some categories, it is more convenient here to introduce another classification: Denote the formal normal form by $\Delta^{*}=A_{0}(h)+x A_{1}(h)$, where $A_{0}(h)=A_{0 k} h^{k}+\ldots, A_{1}(h)=$ $A_{1 l} h^{l}+\ldots$ with $A_{0 k}, A_{1 l} \neq 0$. If one of the series vanishes, we formally put $k=\infty$ or $l=\infty$, respectively. Then as the first case, we consider the case that $l$ is smaller than $k$, in the second case $k \leqslant l$. The first case includes the non-degenerate case. Here we present the following results:

TheOREM 1.4. - In the first case, $A_{1}$ can be reduced to $A_{1 l} h^{l}$ and the power series $U(x, y)=\sum_{r, s} b_{r s} x^{r} y^{s}$ and $A_{0}(h)=\sum_{m \geqslant k} A_{0 m} h^{m}$ are Gevrey of order $\alpha=1 /(6 l+1)$ in the weighted degree; i.e. there exist $K, A>0$ such that for all $r, s, m$

$$
\left|A_{m}\right| \leqslant K A^{6 m \alpha} \Gamma(6 m \alpha+1), \quad\left|b_{r s}\right| \leqslant K A^{(2 r+3 s) \alpha} \Gamma((2 r+3 s) \alpha+1) .
$$

Moreover, the power series $A_{0}\left(t^{6}\right)$ is summable and generically, the above Gevrey order is optimal. In the second case, $A_{0}$ can be reduced to $A_{0 k} h^{k}$ and $U(x, y)$ and $A_{1}(h)$ are Gevrey of order $1 /(3 k+3 l)$ in the weighted degree. Moreover, the power series $A_{1}\left(t^{6}\right)$ is summable and generically, the above Gevrey order is optimal.

The idea of the proof is given in section 3 . We would like to mention that the theory presented here is somewhat analogous to that of J. Martinet and J.-P. Ramis ([MR83]); in the resonant saddle case, $Y^{2}-X^{3}$ is replaced by $Y^{2}-X^{2}$, their normal form is polynomial and in general, the transformation $U$ is divergent but summable. Observe that in our framework, the divergence is introduced by the normal form (recall that formal equivalence of analytic equations of the form $(\Delta)$ implies analytic equivalence) whereas in their case, only the normalising transformation is (in general) divergent. 


\section{The non-degenerate case}

\subsection{Numerical results}

[CMT01] established a specific algorithm derived from F. Loray's work ([Lo99]) in order to determine the Gevrey character of the normal form and the normalizing transformation. They first presented a "pattern recognition" algorithm for the growth rate of the coefficients. This algorithm will be part of a general procedure called Gevreytiseur to establish the numerical Gevrey character of a given series.

Let $S(X)=\sum_{n \geqslant 0} a_{n} X^{n} \in \mathbb{C}[[X]]$. First, the procedure consists in studying the sequences $\left(\frac{a_{n+1}}{a_{n}}\right)_{n \in \mathbb{N}}$ and $\left(a_{n} X^{n}\right)_{n \in \mathbb{N}}$ for certain $X$ in order to determine the radius of convergence of the power series. When the radius of convergence is zero, [CMT01] study the Gevrey character (cf. [Gev18, Ram78]) of the power series.

If a power series is Gevrey of order $1 / p$ and of type $A$, then its coefficients satisfy the following estimate: $a_{n} \leqslant C A^{n / p} \Gamma\left(\rho+\frac{n}{p}\right)$ where $C$ and $\rho$ are positive constants. Often, it seems reasonable to conjecture (cf. [CK99]) that the coefficients $a_{n}$ are asymptotically equivalent to an expression of the above type:

$$
a_{n} \sim C A^{n / p} \Gamma\left(\rho+\frac{n}{p}\right) .
$$

Sequences with a behavior like (2.1) are characterized by the following lemma:

Lemma 2.1. - Let $C>0, A>0, \rho>0$ and $X>0$. The sequence $n \rightarrow C A^{n / p} \Gamma\left(\rho+\frac{n}{p}\right) X^{n}$ is first decreasing, then increasing. The minimum is attained for

$$
N=\left[\frac{p}{A X^{p}}\right]
$$

and this minimum is $K X^{\xi} e^{-X^{p} / A}$ with some $K>0$ and $\xi=\min \left(\frac{1}{p}-\rho+\right.$ $\left.\frac{1}{2}, 0\right)$.

The first method of [CMT01] tests the behavior of $a_{n} X^{n}$ for several of $X$. If they observe the phenomenon described in Lemma 2.1, then they note the index $n=N$ for which $a_{n} X^{n}$ becomes minimal. A rough estimate of the Gevrey order and type then follows from relation (2.2). 
Then applying the method of least squares to $\left(\log a_{n}\right)_{n \in \mathbb{N}}$, they note that the relation (2.1) can also be written as $a_{n} \sim(n !)^{\alpha} B^{n} n^{\beta} M$. Thus the new constants are related to the previous ones by the following relations:

$$
\alpha=\frac{1}{p} ; B=\left(\frac{A}{p}\right)^{1 / p} ; \beta=\rho-\frac{1+p}{2 p} ; M=C p^{\frac{1}{2}-\rho}(2 \pi)^{\frac{p-1}{2 p}} .
$$

The problem consists in determining constants $\alpha, B, \beta$ and $M$ such that the curve $\alpha \ln (n !)+n \ln (B)+\beta \ln (n)+\ln (M)$ is as close as possible to the curve $\ln \left(a_{n}\right)$. The method of least squares is a convenient way of doing this.

Finally interpolating by spline functions, [CMT01] calculate the constants $C, A, \rho$ and $p$ by using spline functions: the value $\ln \left(a_{n}\right)$ is approximated by a spline cubic function $\sigma(n)$ using all $a_{i}$ (for details, see [CMT01]).

In several examples, the study of [CMT01], based on 35 coefficients of $\Delta^{*}$, leads us to conjecture that if the power series $\Delta(x, y)$ contains the monomial $x$ and is convergent, then the normal form $\Delta^{*}=x+h A_{0}(h), h=$ $y^{2}-x^{3}, A_{0} \in \mathbb{C}[[h]]$, is generically Gevrey of order 6 and type $A$ close to 0.18 .

In the present work, we sketch a proof of this conjecture (see Theorem 2) and generalize it to the degenerate case. Observe that a formal power series $\sum_{m \geqslant 0} a_{m} h^{m} \in \mathbb{C}[[h]]$ is Gevrey of order 6 and type $A$ if and only if the power series $\sum_{m \geqslant 0} a_{m} t^{6 m} \in \mathbb{C}[[t]]$ is Gevrey of order 1 and type $A$. The following subsection are devoted to a sketch of our proof.

\subsection{Transformation of the coordinates}

The solution of the unperturbed system suggests the following coordinate transformation

$$
\left\{\begin{array}{l}
x=q(s) t^{2} \\
y=q^{\prime}(s) t^{3}
\end{array}\right.
$$

where $q$ is an elliptic function verifying $q^{\prime 2}=q^{3}+1, q(0)=\infty$. Precisely, $q=4 \mathcal{P}$ where $\mathcal{P}$ is the Weierstrass $\mathcal{P}$-function with parameters $g_{2}=0$ and $g_{3}=-1 / 16$ ([AS64]). Observe that $h=y^{2}-x^{3}=t^{6}$. Each point $(x, y)$ outside the cusp $y^{2}=x^{3}$ corresponds to 6 points $(s, t)$ where $s$ is an element of the hexagon $\mathcal{H}$ defined by the first six zeroes of $q: \mathcal{H}$ is the hexagon with vertices $\rho^{j} c, j=0, \ldots, 5$ where $c=2 \int_{0}^{1}\left(1-t^{3}\right)^{-1 / 2} d t \simeq 2.80436, \rho=e^{\frac{\pi}{3} i}$. 
With the notation

$$
\begin{aligned}
\tilde{U}(s, t) & =(\mathcal{C} U)(s, t)=U\left(q(s) t^{2}, q^{\prime}(s) t^{3}\right) \\
D(s, t) & =(\mathcal{C} \Delta)(s, t) \\
D^{*}(s, t) & =\left(\mathcal{C} \Delta^{*}\right)(s, t)=q(s) t^{2}+t^{6} A_{0}\left(t^{6}\right)
\end{aligned}
$$

the transformation equation (1.2) is simplified to

$$
\left(\tilde{U}+t \tilde{U}_{t}\right) D^{*}=D(s, t \tilde{U})-2 t \tilde{U}_{s}
$$

where $\tilde{U}_{t}=\frac{\partial \tilde{U}}{\partial t}, \tilde{U}_{s}=\frac{\partial \tilde{U}}{\partial s}$. This follows from $2 t \tilde{U}_{s}=\mathcal{C}\left(2 y U_{x}+3 x^{2} U_{y}\right)(s, t)$, $t \tilde{U}_{t}=\mathcal{C}\left(2 x U_{x}+3 y U_{y}\right)(s, t)$ and $D(s, t \tilde{U})=\mathcal{C}\left(\Delta\left(x U^{2}, y U^{3}\right)\right)(s, t)$.

We are looking for solutions in $s$ and $t$ of this equation with certain symmetry properties that can be expressed in terms of power series in $x$ and $y$. Let us introduce the following $\mathbb{C}$-vector spaces: $H_{m}$ the space of homogeneous polynomials $\sum_{2 k+3 l=m} a_{k l} x^{k} y^{l}$ of weighted degree $m$ and $\mathcal{E}_{m}$ the space of meromorphic functions on $\mathbb{C}$ that are $p_{0^{-}}$and $p_{1}$-periodic $\left(p_{0}=\right.$ $2 i a$ and $p_{1}=2 i \rho a$, where $a=\int_{1}^{\infty}\left(t^{3}-1\right)^{-1 / 2} d t \simeq 2.42865$, are fundamental periods of $q$ ), whose only pole inside the hexagon $\mathcal{H}$ is 0 , of order $\leqslant m$, and such that $f(\rho s)=\rho^{-m} f(s)$.

The coordinate change $(\mathcal{C})$ induces a bijection $I$ between $H_{m}$ et $\mathcal{E}_{m}$ by $I(f)(s) t^{m}=\mathcal{C}(f)(s, t)$. Let $\mathcal{S}=\left\{\sum_{m=0}^{\infty} f_{m}(s) t^{m} / f_{m} \in \mathcal{E}_{m}\right\}$ and $\mathcal{S}_{n}=$ $\left\{\sum_{m=n}^{\infty} f_{m}(s) t^{m} / f_{m} \in \mathcal{E}_{m}\right\}(\mathcal{S}$ is the set of all formal power series in $t$ whose coefficients are elliptic functions of $s$ which can be expressed in $x$ and $y$ ).

Using the above notation, given $D \in q(s) t^{2}+\mathcal{S}_{6}$, we are looking for a solution $\tilde{U} \in 1+\mathcal{S}_{5}, D^{*}=q(s) t^{2}+A_{0}\left(t^{6}\right)$ of $(2.5)$. So let:

$$
\begin{aligned}
\tilde{U}(s, t) & =1+t W(s, t) \\
D(s, t) & =q(s) t^{2}+t^{2} E(s, t) \\
D^{*}(s, t) & =q(s) t^{2}+t^{2} E^{*}(s, t) .
\end{aligned}
$$

Then equation (2.5) becomes

$$
2 W_{s}+t^{2} q(s) W_{t}+E^{*}=q(s) t^{2} W^{2}-2 t W E^{*}-t^{2} W_{t} E^{*}+E(s, t(1+t W))(1+t W)^{2} .
$$

We now rewrite equation (2.2) in the Borel plane (see Appendix 4.1 for details). We apply the formal Borel transformation $\hat{\mathcal{B}}_{1}$ with respect to $t$, defined by $\hat{\mathcal{B}}_{1}\left(t^{n}\right)=\frac{\tau^{n-1}}{(n-1) !}$ for $n \geqslant 1$, to all the preceding power series (cf. figure 1). 
Divergence and summability of normal forms of systems of differential equations

$$
\begin{aligned}
& x=q(s) t^{2} \\
& y=q^{\prime}(s) t^{3} \\
& (x, y) \\
& h=y^{2}-x^{3} \\
& t^{6} \\
& \underset{\widehat{\mathcal{B}}_{1}}{\stackrel{\widehat{\mathcal{L}}_{1}}{\longrightarrow}} \quad(s, \tau) \\
& \text { U } \\
& \begin{array}{c|c}
\tilde{U}=1+t W & W
\end{array} \\
& \begin{array}{c|l}
D=q(s) t^{2}+t^{2} E & E \\
D^{*}=q(s) t^{2}+t^{2} E^{*} & E^{*}
\end{array}
\end{aligned}
$$

Figure 1. - Dictionary of the change of variables.

Precisely, let $E(s, t(1+t W))(1+t W)^{2}=\sum_{n=0}^{\infty} F_{n}(s, t) t^{n} W^{n}$ and

$$
\begin{aligned}
\mathbf{W}(s, \tau) & =\hat{\mathcal{B}}(W(s, t)) \\
\mathbf{E}^{*}(\tau) & =\hat{\mathcal{B}}\left(E^{*}(s, t)\right) \\
\mathbf{F}_{n}(s, \tau) & =\hat{\mathcal{B}}\left(F_{n}(s, t)\right) .
\end{aligned}
$$

Using properties of the Borel transform, in particular $\hat{\mathcal{B}}_{1}\left(t^{2} \frac{\partial}{\partial t} f(t)\right)$ $=\tau \hat{\mathcal{B}}_{1}(f(t))$, equation (2.6) can be written as follows in the Borel plane:

$$
\mathcal{L}\left(\mathbf{W}, \mathbf{E}^{*}\right):=2 \mathbf{W}_{s}+q(s) \tau \mathbf{W}+\mathbf{E}^{*}=\mathcal{G}\left(\mathbf{W}, \mathbf{E}^{*}\right),
$$

where

$$
\begin{gathered}
\mathcal{G}\left(\mathbf{W}, \mathbf{E}^{*}\right)(s, \tau):=q(s) \tau * \mathbf{W} * \mathbf{W}-2 \mathbf{W} * \mathbf{E}^{*}-(\tau \mathbf{W}) * \mathbf{E}^{*} \\
+\mathbf{F}_{0}+\sum_{n=1}^{\infty} \mathbf{F}_{n} * \tau^{n-1} * \mathbf{W}^{* n} /(n-1) !
\end{gathered}
$$

where $*$ denotes the convolution product with respect to $\tau$. The restrictions are the following: Given are $\tau^{3} \mathbf{F}_{n} \in \mathcal{S}_{\max (n, 6)}$ and we are looking for $\mathbf{E}^{*}(\tau) \in$ $\tau^{3} \mathbb{C}\left[\left[\tau^{6}\right]\right], \tau^{2} \mathbf{W} \in \mathcal{S}_{5}$. For a summary of all our transformations and the corresponding equations, see figure 3 in the Appendix 4.2.

\subsection{The linearized problem}

We consider the linearization of $(2.7)$ :

$$
\begin{gathered}
2 \mathbf{W}_{s}+q(s) \tau \mathbf{W}+\mathbf{E}^{*}=\mathbf{G} . \\
-501-
\end{gathered}
$$


Given $\tau^{3} \mathbf{G} \in \mathcal{S}_{6}$, we have to find $\tau^{2} \mathbf{W} \in \mathcal{S}_{5}, \mathbf{E}^{*}(\tau) \in \tau^{3} \mathbb{C}\left[\left[\tau^{6}\right]\right]$ verifying this linear ordinary differential equation with a parameter $\tau$. We solve it by method of variation of constants. The "constant" of integration and $\mathbf{E}^{*}$ are uniquely determined by the conditions (in particular $\mathbf{W}$ has to be a single valued function of $s$ ). We find

$$
\mathbf{W}(s, \tau)=e^{-\tau I(s) / 2} \int_{\infty}^{s} e^{\tau I(\sigma) / 2}\left(\mathbf{G}(\sigma, \tau)-\mathbf{E}^{*}(\tau)\right) d \sigma
$$

where $I$ is an antiderivative of $q$, and

$$
\begin{gathered}
\mathbf{E}^{*}(\tau)=\frac{1}{\mathbf{R}(\tau)} \operatorname{res}\left(e^{\tau I(\sigma) / 2} \mathbf{G}(\sigma, \tau), \sigma=0\right) \\
\text { with } \quad \mathbf{R}(\tau)=\operatorname{res}\left(e^{\tau I(\sigma) / 2}, \sigma=0\right) .
\end{gathered}
$$

In (2.9), the path of integration is from $\infty$ to $s$ avoiding the poles of $q$ and such that $\operatorname{Re}(\tau I(\sigma))$ tends to $-\infty$ as $\sigma \rightarrow \infty$.

The zeroes of the function $\mathbf{R}$ of (2.10) are important because they introduce the singularities of $\mathbf{E}^{*}$ (and $\mathbf{W}$ ) in the Borel plane.

\subsection{Zeroes of the function $R$}

We study the zeroes of the function

$$
\mathbf{R}(\tau)=\operatorname{res}\left(e^{\tau I(s) / 2}, s=0\right)=\frac{1}{2 \pi i} \int_{\mathcal{H}} e^{\tau I(\boldsymbol{s}) / 2} d s .
$$

Here, the function $I(s)$ is the unique antiderivative of $q(s)$ without constant term; $I(s)=-4 \zeta(s)$ where $\zeta(s)$ denotes the Weierstrass zeta function with parameters $g_{2}=0$ and $g_{3}=-1 / 16$ ([AS64]). $I$ is a pseudo-periodic function. Its (simple) zeroes are $p_{0} / 2, p_{1} / 2, \ldots, p_{5} / 2$ and its saddle-points are the zeroes of $I^{\prime}=q$, that is, the vertices $\rho^{j} c, j=0, \ldots, 5$ of the hexagon $\mathcal{H}$.

Obviously, $\mathbf{R}$ is an entire function. As $I(\rho s)=\rho^{-1} I(s)$, we have $\mathbf{R}(\rho \tau)=$ $\rho \mathbf{R}(\tau)$ and the coefficients of $\mathbf{R}$ are real like those of $q$ and $I$. So

$$
\mathbf{R}(\tau)=\tau \sum_{m=0}^{\infty} R_{m} \tau^{6 m} \text { with } R_{m} \in \mathbb{R}, \forall m \geqslant 0 .
$$

If $\tau$ is a zero of $\mathbf{R}$, then $\rho^{\nu} \tau$ and $\rho^{\nu} \bar{\tau}, \nu=0, \ldots, 5$ are zeroes of $R$, too. We have the symmetries $\mathbf{R}\left(\rho^{\nu} \tau\right)=\rho^{\nu} \mathbf{R}(\tau), \mathbf{R}\left(\rho^{\nu} \bar{\tau}\right)=\rho^{\nu} \overline{\mathbf{R}(\tau)}, \nu=0, \ldots, 5$. 
Divergence and summability of normal forms of systems of differential equations

The first terms of the series of $R$ are

$$
\mathbf{R}(\tau)=-2 \tau-\frac{1}{12600} \tau^{7}-\frac{17}{856215360000} \tau^{13}-\ldots
$$

Lemma 2.2. - The function $\mathbf{R}$ has exponential growth as $|\tau| \rightarrow \infty$; more precisely

$$
|\mathbf{R}(\tau)| \leqslant C \exp (D|\tau|), \text { where } C=\frac{3 c}{\pi} \leqslant 2.68, D=\frac{I(-c)}{2} \leqslant 0.863 .
$$

Moreover, we can localize the zeroes of the function $\mathbf{R}$ :

THEOREM 2.3. - The zeroes of the function $\mathbf{R}$ (other than $\tau=0$ ) are on the rays $\arg \tau=(2 l+1) \frac{\pi}{6}, l=0, \ldots, 5$ and form six sequences $m_{k} e^{(2 l+1) \pi i / 6}, k \in \mathbb{N}, l=0, \ldots, 5$. One has $\left|m_{0}-5.4204\right|<0.0002$ and $\left|m_{k}-t_{k}\right|<12 t_{k}^{-3}$ with $t_{k}=\frac{\pi}{I(-c)}(3+4 k)$ if $k \geqslant 1$; here $I(-c)=$ $2 \int_{0}^{1} t\left(1-t^{3}\right)^{-1 / 2} d t \simeq 1.72474$.

Sketch of the proof: First, we study the zeroes near the origine. We have the result:

THEOREM 2.4. - The function $\mathbf{R}$ has exactly 7 simple zeroes in the disk $|\tau|<10.6$. They are $\tau=0$ and $\tau=\rho^{\nu} i m_{0}, \nu=0, \ldots, 5$, where $m_{0}$ is real and $\left|m_{0}-5.4204\right|<0.0002$. Thus $\frac{1}{m_{0}} \simeq 0.1845$.

The zeroes of $\mathbf{R}$ satisfying $|\tau|>10.6$ are less easily located; we use an asymptotic method: since $\mathbf{R}(\tau)$ is given by an integral formula with an exponential term, we apply the "saddle point method" to determine precise asymptotic estimates, i.e. we use Laplace's method with error bounds ([Olv74]) for large $\tau$.

Remark 2.5. - The modulus of the first zeroes of $\mathbf{R}$ is almost equal to 5.4204. Now, we are going to prove that the first zeroes of $\mathbf{R}$ effectively are the first singularities of $\mathbf{E}^{*}$; so we will conclude that $\Delta^{*}$ is divergent, Gevrey of order 6 (in $t^{6}$ ) and with a type equal to $\frac{1}{5.4204} \simeq 0.1845$. This value of the type was obtained numerically in [CMT01]. 


\subsection{The non-linear problem}

As the linear differential equation has a unique solution $\left(\mathbf{W}, \mathbf{E}^{*}\right)$ satisfying the restrictions, we obtain two linear operators $\mathcal{W}(\mathbf{G}):=\mathbf{W}$, $\mathcal{E}(\mathbf{G}):=\mathbf{E}^{*}$.

In order to find a solution of (2.7), it is sufficient to solve the fixed point equation $\mathbf{G}=\mathcal{G}(\mathcal{W}(\mathbf{G}), \mathcal{E}(\mathbf{G}))$ in $\tau^{-3} \mathcal{S}_{6}$, i.e. to solve

$$
\mathbf{G}=\mathcal{F}(\mathbf{G}),
$$

where $\mathcal{F}(\mathbf{G})=q(s) \tau * \mathcal{W}(\mathbf{G}) * \mathcal{W}(\mathbf{G})-2 \mathcal{W}(\mathbf{G}) * \mathcal{E}(\mathbf{G})-(\tau \mathcal{W}(\mathbf{G})) * \mathcal{E}(\mathbf{G})$

$$
+\mathbf{F}_{0}+\sum_{n=1}^{\infty} \mathbf{F}_{n} * \tau^{n-1} * \mathcal{W}(\mathbf{G})^{* n} /(n-1) \text { ! . }
$$

Let $\mathcal{D}=\mathbb{C} \backslash \cup_{\mu=0}^{5} e^{\frac{\pi}{6} i} e^{\mu \frac{\pi}{3} i}\left[m_{0}, \infty[\right.$.

THEOREM 2.6. - There exists a unique analytic solution $\mathbf{G}:(\mathbb{C} \backslash \mathcal{R}) \times$ $\mathcal{D} \rightarrow \mathbb{C}$ of (2.16). It is $\mathcal{R}$-periodic, symmetric: $\mathbf{G}(\rho s, \rho \tau)=\rho^{-3} \mathbf{G}(s, \tau)=$ $-\mathbf{G}(s, \tau)$ and $\mathbf{G}(s, \tau) \in \tau^{-3} \mathcal{S}_{6}$.

As a consequence, $\mathbf{G}$ and thus also $\mathcal{W}(\mathbf{G})=\mathbf{W}, \mathcal{E}(\mathbf{G})=\mathbf{E}^{*}$ have convergent power series representations at $\tau=0$, hence the formal solution $\left(\Delta^{*}, U\right)$ is Gevrey 1.

We also need a statement concerning the growth of $\mathbf{G}(s, \tau)$ as $\tau \rightarrow \infty$ along rays in $\mathcal{D}$.

Theorem 2.7. - Consider $\theta \in \mathbb{R} \backslash\left(\frac{\pi}{6}+\frac{\pi}{3} \mathbb{Z}\right)$. For sufficiently small $\delta>0$, there exists $K, M>0$ such that $|\mathbf{G}(s, \tau)| \leqslant M \exp (K|\tau|)$ for all $s, \tau \in \mathbb{C}$ with $\operatorname{dist}(s, \mathcal{R})>\delta$ and $|\arg \tau-\theta|<\delta$.

Here, we only give a rough idea of the proof of Theorem 2.7. We introduce a certain subset $\mathcal{A}_{\theta}$ of $\mathbb{C}$ of $s$ that can be joined to $\infty$ by a path $\gamma(\sigma),-\infty<$ $\sigma \leqslant 0$ on which $\operatorname{Re}\left(e^{\theta i} I(\gamma(\sigma))\right)$ increases. Then we obtain an inequality containing convolutions for

$$
f(r)=\sup \left\{|\mathbf{G}(s, \tau)|\left|s \in \mathcal{A}_{\theta},\right| \arg \tau-\theta|<\delta,| \tau \mid \leqslant r\right\} .
$$

Following an idea of B.L.J. Braaksma and W. Walter, we show, using the Laplace transform, that the corresponding equation has a solution having at most exponential growth and that it is a majorant of $f(r)$. This shows 
Divergence and summability of normal forms of systems of differential equations

the statement of the theorem for $s \in \mathcal{A}_{\theta}$. Finally we extend this statement to $s$ outside $\mathcal{A}_{\theta}$.

Observe that this theorem implies that also $\mathbf{E}^{*}=\mathcal{E}(\mathbf{G})$ is at most of exponential growth as $\tau \rightarrow \infty$. This proves the 1-summability of $A_{0}\left(t^{6}\right)$ stated in Theorem 1.3. We obtain also the 1-summability of $\tilde{U}(s, t)=(\mathcal{C} U)(s, t)$ with respect to $t$.

\subsection{Divergence}

In order to show the divergence of the formal solution in the generic case, we show that the Borel transform $\mathbf{E}^{*}(\tau)$ has a singularity at $\tau=\tau_{j}=$ $m_{0} \exp \left(\frac{\pi}{6} i+j \frac{\pi}{3} i\right), j=0, \ldots, 5$. We establish this fact in a way similar to the preceding proof, but we work with a different function space (a very simplified version of the resurgent functions of J. Ecalle [Eca85]).

We consider the subset $\tilde{D}$ of the universal covering $\hat{D}$ of $\{\tau \in \mathbb{C}|| \tau \mid<$ $\left.m_{0}+1\right\} \backslash\left\{\tau_{0}, \ldots, \tau_{5}\right\}$ of all points that can either be joined (in $\hat{D}$ ) to 0 by a segment or whose distance from one of the $\tau_{j}, j=0, \ldots, 5$ is smaller than 1 . We write this decomposition $\tilde{D}=\check{D} \cup \cup_{j=0}^{5} B_{j}$. Let $\mathcal{O}$ the Banach space of all holomorphic functions $\mathbf{G}: \mathcal{C}_{\delta} \times \tilde{D} \rightarrow \mathbb{C}$ bounded on $\mathcal{C}_{\delta} \times \check{D}$ that can be written as

$\mathbf{G}(s, \tau)=\alpha_{j}^{\mathbf{G}}(s, \tau)+\beta_{j}^{\mathbf{G}}(s, \tau)\left(\tau-\tau_{j}\right) \log \left(\tau-\tau_{j}\right), 0<\left|\tau-\tau_{j}\right|<1,0 \leqslant j \leqslant 5$, where $\alpha_{j}^{\mathbf{G}}$ and $\beta_{j}^{\mathbf{G}}$ are bounded holomorphic functions on $\mathcal{C}_{\delta} \times\left\{\left|\tau-\tau_{j}\right|<1\right\}$.

First we show that (2.16) has a unique solution $\mathbf{G}$ in the closed subspace $\mathcal{O}_{6,6}$ of all $\mathbf{H} \in \mathcal{O}$ such that $\tau^{-6} \mathbf{H}(s, \tau) \in \mathcal{O}$ and all $\left(\tau-\tau_{j}\right)^{-6} \beta_{j}^{\mathbf{H}}(s, \tau)$ are also bounded. By (2.10), there exists a constant $a_{0} \in \mathbb{C}$ such that $\mathbf{E}^{*}-$ $\sum_{j=0}^{5} \frac{a_{0} \rho^{-2 j}}{\tau-\tau_{j}} \in \mathcal{O}_{6,5}$. Then we show that $a_{0}$ depends analytically upon the coefficients of $\Delta$ and by considering the example $\Delta=x+\varepsilon y, \varepsilon \neq 0$ small, we show that $a_{0}$ is a non trivial function of the coefficients of $\Delta$. This proves the divergence of $E^{*}$ (and hence of $\Delta^{*}$ and consequently $U$ ) in the generic case and yields the function $Q$ of the theorem. Moreover, in the case of $a_{0} \neq 0$, the type is equal to $\frac{1}{\left|\tau_{0}\right|}$.

Remark 2.8. - One might hope to achieve a convergent "prenormal" form by allowing two full series $A_{0}(h), A_{1}(h)$. This does, unfortunately, not help: a "final" transformation $x \leftarrow x U(h)^{2}, y \leftarrow y U(h)^{3}$ with convergent $U(h)$ reduces such a convergent prenormal form to a convergent normal form! 


\section{The degenerate cases}

\subsection{Numerical results}

In [CMT01], a second group of tests concerned power series which do not contain the monomial $x$ (degenerate cases). The following two cases were considered:

a) The power series $\Delta(x, y)$ is of the form: $b x^{3}+\sum_{p+q \geqslant 2, p \neq 0,2 p+3 q \geqslant 7} x^{p} y^{q}$ with some $b \neq 0$. Here the (modified) formal normal form is

$$
\Delta^{*}=b x^{3}+x A_{1}\left(x^{3}\right)
$$

b) The power series $\Delta(x, y)$ is of the form: $C x^{4}+\sum_{p+q \geqslant 3, p \neq 0,2 p+3 q \geqslant 9} x^{p} y^{q}$ with some $C \neq 0$. Here the (modified) formal normal is

$$
\Delta^{*}=C x^{4}+x^{3} A_{0}\left(x^{3}\right)
$$

For each case [CMT01] used a specific algorithm. Contrarily to their previous tests, the curve $n \rightarrow \ln \left(a_{n}\right)$ here was not regular. It was not possible to estimate the Gevrey order $\frac{1}{p}$ consistently even with the method of least squares and the results were very different depending on whether all the coefficients of $\Delta^{*}$ were considered in the curve fitting or only part of these. Briefly, [CMT01] did not obtain a precise conclusion.

Later, the calculations were redone for simpler $\Delta$, namely $\Delta(x, y)=$ $x h+y^{3}$ and $\Delta(x, y)=h+x h+y^{3}$. These tests allowed to conjecture the results stated in Theorem 1.4 .

\subsection{Idea of the proof}

In the first case, the proof is very much like the one for the non-degenerate case presented above. First, new coordinates are introduced by $(\mathcal{C})$ and the principal linear part is isolated. Instead of equation (2.5), we obtain

$$
2 W_{s}+q(s) t^{6 l+2} W_{t}+E^{*}=\ldots
$$

and this implies that the appropriate Borel transform is the one that essentially maps $t^{6 l+2} \frac{\partial}{\partial t}$ to the multiplication by a power of $\tau$. Precisely, this means that we have to use $f \mapsto \tau^{1-p} \widehat{\mathcal{B}}_{p}\left(t^{p-1} f(t)\right)$ with $p=6 l+1$. The remaining steps of the proof are analogous to the ones given in section 2 .

In the second case, the result is somewhat surprising as the optimal Gevrey order does not only depend upon the first non-vanishing term $A_{0 k} h^{k}$ 
of the formal normal form but also of the first non-vanishing term of the other series $A_{1}(h)=A_{1 l} h^{l}+\ldots$. This can be interpreted in terms of the variables introduced in $(\mathcal{C})$ : in the new variables, $x=q(s) t^{2}$ introduces a pole in the $s$-plane that implies that the function $\mathbf{R}(\tau)$ of $(2.11)$ has nontrivial zeroes. If the leading term is $h=t^{6}$, the analog of the function $\mathbf{R}(\tau)$ equals simply $C \tau$ and introduces no singularities in the Borel plane. It was also surprising for us that the change of variables $(\mathcal{C})$ successful in the non-degenerate case is inappropriate in the second case. In the sequel we discuss the ideas for the simplest subcase of the second case, i.e. $k=l=1$ and $A_{01}=A_{11}=1$. In this case, the variables we use are $h$ and $x$ - the variable $y$ is eliminated.

We proceed as follows. The main linear part of the transformation equation is isolated on the left hand side; thus (1.2) becomes

$$
2 y W_{x}+3 x^{2} W_{y}+(h+x h)\left(2 x W_{x}+3 y W_{y}\right)+x E^{*}(h)=\ldots
$$

where $U(x, y)=1+W(x, y), \Delta^{*}=h+x h+x E^{*}(h)$ and the dots indicate nonlinear and other lower order terms that are not specified; in a complete proof these terms would appear on the right hand side in equations analogous to (2.6) and (2.7) and the fixed point theorem would be applied. Here, we only indicate the treatment of the analog of the linear equation (cf. subsection 2.4).

Using $y^{2}=h+x^{3}$, every formal series $f(x, y)$ can be written in the form $f(x, y)=f^{(0)}(x, h)+y f^{(1)}(x, h)$; here $f^{(0)}$ and $f^{(1)}$ are uniquely determined. In this way, we can write (3.1) as a system of two equations for $W^{(0)}$ and $W^{(1)}$.

$$
\begin{aligned}
2\left(h+x^{3}\right) W_{x}^{(1)}+3 x^{2} W^{(1)}+(h+x h)\left(2 x W_{x}^{(0)}+6 h W_{h}^{(0)}\right) & \\
+x E^{*}(h) & =\ldots \\
2 W_{x}^{(0)}+(h+x h)\left((3+2 x) W_{x}^{(1)}+6 h W_{h}^{(1)}\right) & =\ldots .
\end{aligned}
$$

Here the terms $2 h W_{x}^{(1)}$ and $(h+x h) x W_{x}^{(0,1)}$ are less important and can also be put on the right hand side. Thus we study the simplified system

$$
\begin{aligned}
2 x^{3} W_{x}^{(1)}+3 x^{2} W^{(1)}+6(1+x) h^{2} W_{h}^{(0)}+x E^{*}(h) & =\ldots \\
2 W_{x}^{(0)}+6(1+x) h^{2} W_{h}^{(1)} & =\ldots .
\end{aligned}
$$

The appearence of $h^{2} \frac{\partial}{\partial h}$ indicates that the Borel transform of order 1 with respect to $h$ is the right choice. Let denote $w_{j}(x, \tau)=\hat{\mathcal{B}}_{1}\left(W^{(j)}(x, h)\right)$ and 
$D^{*}(\tau)=\hat{\mathcal{B}}_{1}\left(E^{*}(h)\right)$ Then the equation becomes

$$
\begin{aligned}
2 x^{3} w_{1}^{\prime}+3 x^{2} w_{1}+6 \tau(1+x) w_{0}+x D^{*}(\tau) & =D_{0}(x, \tau) \\
2 w_{0}^{\prime}+6 \tau(1+x) w_{1} & =D_{1}(x, \tau) .
\end{aligned}
$$

From now on $\tau$ is regarded as a parameter and thus differentiation with respect to $x$ is simply denoted by '; the right hand sides have been given names now - keep in mind that they contain nonlinear terms...

Next, we introduce $y_{0}=w_{0}$ and $y_{1}=x^{3 / 2} w_{1}$; they satisfy

$$
\begin{aligned}
2 x^{3 / 2} y_{1}^{\prime}+6 \tau(1+x) y_{0}+x D^{*}(\tau) & =D_{0}(x, \tau) \\
2 x^{3 / 2} y_{0}^{\prime}+6 \tau(1+x) y_{1} & =x^{3 / 2} D_{1}(x, \tau) .
\end{aligned}
$$

As $y_{0}$ and $D_{0}$ only contain powers of $x$ with integer exponents and $y_{1}$ and $x^{3 / 2} D_{1}(x, \tau)$ only contain powers of $x$ with exponents of the form "integer + $1 / 2$ ", the above equations can be added without loss of information. Denote $z=y_{0}+y_{1}, f(x, \tau)=D_{0}(x, \tau)+x^{3 / 2} D_{1}(x, \tau)$. Then we obtain

$$
2 x^{3 / 2} z^{\prime}+6 \tau(1+x) z+x D^{*}(\tau)=f(x, \tau) .
$$

The last change of variables is $x=t^{2}$; we keep the names of the functions for the sake of simplicity, only differentiation with respect to $t$ will be denoted by a dot.

$$
t^{2} \dot{z}+6 \tau\left(1+t^{2}\right) z+t^{2} D^{*}(\tau)=f(t, \tau) .
$$

This last equation can again be solved by variation of constants.

$$
z(t, \tau)=e^{6 \tau\left(\frac{1}{t}-t\right)}\left(C+\int^{t} e^{-6 \tau\left(\frac{1}{s}-s\right)}\left(s^{-2} f(s, \tau)-D^{*}(\tau)\right) d s\right) .
$$

Again, we need that $z$ is a single valued function of $t$; hence the residue of the integrand must vanish. Thus $D^{*}$ is determined by the formula

$$
D^{*}(\tau)=\operatorname{res}_{s=0}\left(e^{-6 \tau\left(\frac{1}{s}-s\right)}\left(s^{-2} f(s, \tau)\right) / \operatorname{res}_{s=0}\left(e^{-6 \tau\left(\frac{1}{s}-s\right)}\right) .\right.
$$

Here, the denominator happens to be a known special function:

$$
J_{1}(12 \tau)=-\operatorname{res}_{s=0}\left(e^{-6 \tau\left(\frac{1}{s}-s\right)}\right) .
$$

It grows at most exponentially as $\tau \rightarrow \infty$ and has two sequences of zeroes on the positive resp. negative real axis. In order to obtain a function $z(t, \tau)$ analytic at $t=0$, the constant $C$ has to be chosen $C=0$ and the path of 
integration in (3.8) has to be from 0 to $t$ such that $\operatorname{Re}(-\tau / t) \rightarrow \infty$ as $t \rightarrow 0$ along the path. We write

$$
z(t, \tau)=e^{6 \tau\left(\frac{1}{t}-t\right)} \int_{0}^{t} e^{-6 \tau\left(\frac{1}{s}-s\right)}\left(s^{-2} f(s, \tau)-D^{*}(\tau)\right) d s
$$

where $D^{*}$ is determined by (3.9).

As in the non-degenerate case, the zeroes of the denominator of (3.9) generically introduce singularities in the Borel plane which correspond to divergence of Gevrey order 1 in the variable $h$ (or Gevrey order 1/6 in the weighted degree). As before, the summability of the obtained series can be shown. This will be detailed in a subsequent article.

The general second case with $k=l$ is very analogous to the above, only the Borel transform will be for a different Gevrey order. If $k<l$, however, another complication occurs: instead of $x$, the variable $\xi=x h^{l-k}$ has to be used; in order that no negative powers of $h$ appear in the equation when this change of variable is made, all terms of $\Delta$ containing a too high power of $x$ have to be eliminated by a preliminary transformation. Luckily, this can be achieved using a convergent $U(x, y)$. For details, we refer to our forthcoming article.

\section{Appendix}

\subsection{The Borel plane}

We recall some definitions and properties of Gevrey asymptotics. We use the formal Borel transform $\widehat{\mathcal{B}}$ in order to recognize Gevrey power series.

Definition 4.1 ([Gev18, Ram78, RS96]). - For any given $p, A>$ 0 , a formal power series $\widehat{f}(t)=\sum_{n \geqslant 0} a_{n} t^{n} \in \mathbb{C}[[t]]$ is said to be Gevrey of order $1 / p=\alpha$ and type $A$, if there exist two nonnegative numbers $C$ and $\rho$ such that

$$
\forall n, n \geqslant 0, \quad\left|a_{n}\right| \leqslant C A^{n / p} \Gamma(\rho+n / p)=C A^{\alpha n} \Gamma(\rho+\alpha n) .
$$

DEFINITION 4.2. - Let $\widehat{f}(t)=\sum_{n \geqslant 1} a_{n} t^{n}$ be a formal power series. We call formal Borel transform of order $\alpha=1 / p$ of $\widehat{f}$ the power series $\widehat{\mathcal{B}}_{p}(\widehat{f})$ defined by

$$
\begin{aligned}
\widehat{\mathcal{B}}_{p}(\widehat{f})(\tau)= & \sum_{n \geqslant 0} \frac{a_{n+1}}{\Gamma(1+n / p)} \tau^{n} \\
& -509-
\end{aligned}
$$




$$
\begin{array}{ccc}
\widehat{f}(t)=\sum_{n \geqslant 1} a_{n} t^{n} & \longrightarrow & \widehat{\mathcal{B}}_{p}(\widehat{f})(\tau)=\sum_{n \geqslant 1} a_{n} \frac{\tau^{n-1}}{\Gamma\left(\frac{n+p-1}{p}\right)} \\
\uparrow & \mid \\
\text { Gevrey asymptotic expansion } \\
\text { of order } 1 / p \\
\mid \\
\begin{array}{c}
\tilde{f}(t):=\mathcal{L}_{R, p}(\mathbf{f})(t)= \\
\int_{0}^{R} e^{-\tau^{p} / t^{p}} \mathbf{f}(\tau) \frac{\tau^{p-1}}{t^{p-1}} d \tau
\end{array} & \longleftarrow & \mathbf{f}(\tau) \text { analytic on } D(0, R) . \\
t \in S, S \text { sector } & &
\end{array}
$$

Figure 2. - Gevrey asymptotics.

DEFINITION 4.3. - Let $\mathbf{f}(\tau)$ be an analytic function on $D(0, R) R>0$. The truncated Laplace transform of level $p$ of $\mathbf{f}$ is the function

$$
\mathcal{L}_{R, p}(\mathbf{f})(t)=p \int_{0}^{R} e^{-\tau^{p} / t^{p}} \mathbf{f}(\tau) \frac{\tau^{p-1}}{t^{p-1}} d \tau
$$

DEFINITION 4.4. - Let $S$ be an open sector with vertex at the origin. Let $f$ be an analytic function on $S$, let $\widehat{f}(t)=\sum_{n \geqslant 0} b_{n} t^{n} \in \mathbb{C}[[t]]$ be a formal power series and let $A, p$ be positive real numbers. We say that $f$ admits $\widehat{f}(t)$ as an asymptotic expansion of Gevrey order $1 / p$ and of type $A$ as $t \rightarrow 0$ on $S$ if there is a positive constant $\rho>0$, and for every subsector $S^{\prime} \prec S$ there is a constant $C_{S^{\prime}}>0$, such that

$$
\forall t \in S^{\prime}, \forall N \in \mathbb{N}^{*}, \quad\left|f(t)-\sum_{n=0}^{N-1} b_{n} t^{n}\right| \leqslant C_{S^{\prime}} A^{N / p} \Gamma(\rho+N / p)|t|^{N} .
$$

In figure 2, we indicate the most important properties of the Borel and Laplace transforms in connection with formal power series of Gevrey order $1 / p$. Further properties:

1. A formal power series is a Gevrey power series of order $\alpha=1 / p$ if and only if its formal Borel transform of order $\alpha$ is a convergent power series in the Borel plane. 
Divergence and summability of normal forms of systems of differential equations

2. If the power series $\widehat{f}(t)$ is a convergent power series, the power series $\widehat{\mathcal{B}}_{p}(\widehat{f})(\tau)$ has a radius of convergence equal to $+\infty$, for all $p>0$.

3. A power series $\widehat{f}(t)$ is divergent, but of Gevrey order exactly $\alpha=1 / p$ and exact type $A$, if and only if $\widehat{\mathcal{B}}_{p}(\widehat{f})(\tau)$ is a convergent power series in the $\tau$-plane and defines an analytic function $\mathbf{f}$ having at least a singularity. Moreover, the modulus of the first singularity is equal to $1 / A^{\alpha}$ (see $\left.[\mathrm{Ca03}]\right)$.

4. The truncated-Laplace transform of $\mathbf{f}$ defines an analytic function on an open sector whose opening is $\langle\alpha \pi$ and this analytic function has $\widehat{f}$ as asymptotic expansion of Gevrey order $\alpha$ (see [Ca03]).

Next, we recall the definitions of $p$-summability ([Ram80]):

DeFinition 4.5. - Let $p$ be a positive real number and let $d_{\phi}$ be a direction. A power series $\widehat{f} \in \mathbb{C}[[t]]$ is $p$-summable in the direction $d_{\phi}$ if the power series is Gevrey of order $\alpha=1 / p$ and if the sum of the convergent power series $\widehat{\mathcal{B}}_{p}(\widehat{f})(\tau)$ has an analytic continuation $\mathbf{f}(\tau)$ that is holomorphic and has an exponential increasing of order at most $p$ at the infinite on an open sector $V$ in the neighbourhood of $d_{\phi}$. Under these conditions, we say that

$$
f(t)=\mathcal{L}_{\phi, p}(\mathbf{f})(t)=\int_{d_{\phi}} e^{-\tau^{p} / t^{p}} \mathbf{f}(\tau) \frac{\tau^{p-1}}{t^{p-1}} d \tau
$$

is the sum of $\widehat{f}$ in the direction $d_{\phi}$ in the Borel-Laplace sense.

This definition is equivalent to the

Definition 4.6. - Let $d_{\phi}$ be a direction. A formal power series $\widehat{f} \in$ $\mathbb{C}[[t]]$ is said to be $p$-summable in the direction $d_{\phi}$ if there exists an holomorphic function $f$ on a sector $S$, bisected by $d_{\phi}$, with opening $>\pi / p$ and $f$ has $\widehat{f}$ as asymptotic expansion with Gevrey estimates of order $\alpha=1 / p$ on $S$.

Under these conditions, $\widehat{f}$ is a Gevrey power series of order $\alpha$ and the sum $f$ is unique. We will say that $f$ is the sum of $\widehat{f}$ in the direction $d_{\phi}$ in the sense of the $p$-summability.

Definition 4.7. - A formal power series $\widehat{f} \in \mathbb{C}[[t]]$ is said to be $p$ summable if the power series is p-summable in every direction $d$ except a finite number of directions. These singular directions are called anti-Stokes lines. 


\subsection{Summary of the equations in the non-degenerate case}

In this appendix, we condense all the changes of variables we made in the non-degenerate case and the resulting equations into one table; in the left column the corresponding equation numbers in the above text are indicated.

\begin{tabular}{|c|c|}
\hline$(1.2)$ & $\left(U+2 x U_{x}+3 y U_{y}\right) \Delta^{*}=\Delta\left(x U^{2}, y U^{3}\right)-2 y U_{x}-3 x^{2} U_{y}$ \\
\hline$(\mathcal{C})$ & $\left\{\begin{array}{l}x=q(s) t^{2} \\
y=q^{\prime}(s) t^{3}\end{array}\right.$ \\
\hline$(2.4)$ & $\begin{aligned} \tilde{U}(s, t) & =(\mathcal{C} U)(s, t)=U\left(q(s) t^{2}, q^{\prime}(s) t^{3}\right) \\
D(s, t) & =(\mathcal{C} \Delta)(s, t) \\
D^{*}(s, t) & =\left(\mathcal{C} \Delta^{*}\right)(s, t)=q(s) t^{2}+t^{6} A_{0}\left(t^{6}\right)\end{aligned}$ \\
\hline$(2.5)$ & $\left(\tilde{U}+t \tilde{U}_{t}\right) D^{*}=D(s, t \tilde{U})-2 t \tilde{U}_{s}$ \\
\hline$(2.6)$ & $\begin{aligned} \tilde{U}(s, t) & =1+t W(s, t) \\
D(s, t) & =q(s) t^{2}+t^{2} E(s, t) \\
D^{*}(s, t) & =q(s) t^{2}+t^{2} E^{*}(s, t)\end{aligned}$ \\
\hline$(2.6)$ & $\begin{aligned} 2 W_{s}+t^{2} q(s) W_{t}+E^{*} & =q(s) t^{2} W^{2} \\
& -2 t W E^{*}-t^{2} W_{t} E^{*} \\
& +E(s, t(1+t W))(1+t W)^{2}\end{aligned}$ \\
\hline & $\uparrow \widehat{\mathcal{L}}$ \\
\hline$(2.7)$ & $\begin{aligned} 2 \mathbf{W}_{s}+q(s) \tau \mathbf{W}+\mathbf{E}^{*} & =q(s) \tau * \mathbf{W} * \mathbf{W} \\
& -2 \mathbf{W} * \mathbf{E}^{*}-(\tau \mathbf{W}) * \mathbf{E}^{*} \\
& +F_{0}+\sum_{n=1}^{\infty} F_{n} * \frac{\tau^{n-1}}{(n-1) !} * \mathbf{W}^{* n}\end{aligned}$ \\
\hline
\end{tabular}

Figure 3. - Summary.

\section{Bibliography}

[AS64] Abramowitz (M.), Stegun editors (I.A.), Handbook of Mathematical Functions, Dover Press, New York (1964).

[Ca91] Canalis-Durand (M.), Formal expansions of van der Pol equation canard solutions are Gevrey, in Dynamic bifurcations, E. Benoît Ed., Lecture Notes in Math., 1493, 29-39 (1991) ou C. R. Acad. Sci., série I 311, p. 27-30 (1990). 
Divergence and summability of normal forms of systems of differential equations

[Ca03] Canalis-Durand (M.), Gevrey normal form of system of differential equations with a nilpotent linear part, IRMA Lectures in Mathematics and Theoretical Physics. From Combinatories to Dynamical Systems, F. Fauvet and C. Mitschi eds., W. de Gruyter, (2003), 131-162.

[CDG89] Canalis-Durand (M.), Diener (F.), Gaetano (M.), Calcul des valeurs à canard à l'aide de Macsyma, in Mathématiques Finitaires et Analyse Non Standard, Luminy 1985, M. Diener, G. Wallet Eds., Preprint URA 212, U.F.R. de Math., Univ. Paris 7, 4 place Jussieu, 75251 Paris Cedex, France, p. 149-163 (1989).

[CK99] Costin (O.), Kruskal (M.), On optimal truncation of divergent series solutions of nonlinear differential systems; Berry smoothing, Proc. R. Soc. Lond., A 455, p. 1931-1956 (1999).

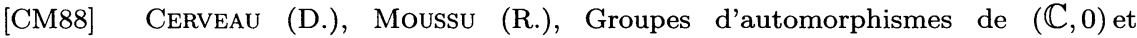
équations différentielles $y d y+\ldots=0$, Publ. Soc. Math., France, 116, p. 459-488 (1988).

[CMT01] Canalis-Durand (M.), Michel (F.), Teisseyre (M.), Algorithms for Formal Reduction of Vector Fields Singularities, Journal on Dynamical and Control Systems, vol. 7, $\mathrm{n}^{\circ}$. 1, p. 101-125 (2001).

[CRSS00] Canalis-Durand (M.), Ramis (J.-P.), Schäfke (R.), SibuYa (Y.), Gevrey solutions of singularly perturbed differential equations, Für Die Reine Und Angewandte Mathematik, Crelles Journal, 518,p. 95-129 (2000).

[CS00] Canalis-Durand (M.), SchäFKe (R.), Caractère Gevrey de la normalisation de Frank Loray pour une certaine équation différentielle nilpotente, Communication in Journées des Equations Différentielles et de Calcul Formel, Lille, p. 16-18 (mars 2000).

[CS03] CANalis-Durand (M.), SchäfKe (R.), On the normal form of a system of differential equations with nilpotent linear part, C. R. Acad. Sci., Sér. I 336, p. 129-134 (2003).

[Eca85] ECALLE (J.), Les fonctions résurgentes. III: L'équation du pont et la classification analytique des objets locaux, Publ. Math. Orsay, Paris, p. 85-05 (1985).

[Gev18] Gevrey (G.), Sur la nature analytique des solutions des équations aux dérivées partielles, Ann. Sci. Ec. Norm. Sup., 3ème série 35, 129-90 (o.c.p 243-304) (1918).

[Lo99] LORAY (F.), Réduction formelle des singularités cuspidales de champs de vecteurs analytiques, Differential Equations, 158 1, p. 152-173 (1999).

[MR83] MARTinet (J.), RAMIS (J.-P.), Classification analytique des équations différentielles non linéaires résonnantes du premier ordre, Ann. Sci. Ec. Norm. Sup., 16, p. 571-621 (1983).

[Olv74] Olver (F.W.J.), Asymptotics and special functions, New-York Academic Press (1974).

[Ram78] Ramis (J.-P.), Dévissage Gevrey, Astérisque (Soc. Math. France), 59-60, p. 173-204 (1978).

[Ram80] Ramis (J.-P.), Les séries $k$-sommables et leurs applications, Analysis, Microlocal Calcul and Relativistic Quantum Theory, Proceedings "Les Houches" 1979, Springer, Lecture Notes in Physics, 126, p. 178-199 (1980).

[RS96] RAMIS (J.-P.), SCHÄFKE (R.), Gevrey separation of fast and slow variables, Nonlinearity, 9, p. 353-84 (1996).

[Tho90] Thomann (J.), Resommation des séries formelles solutions d'équations différentielles linéaires ordinaires du second ordre dans le champ complexe au voisinage de singularités irrégulières, Numer. Math., 58, p. 503-535 (1990). 